\title{
Radionuclide Imaging of the Gut-Brain Axis in Parkinson Disease
}

\author{
Jacob Horsager, Karoline Knudsen, and Per Borghammer \\ Department of Nuclear Medicine and PET, Aarhus University Hospital, Aarhus, Denmark
}

$\mathbf{T}$ he gut-brain axis is a bidirectional communication system between the enteric and central nervous systems, comprising neuronal, hormonal, and immunologic mechanisms. For instance, the autonomic nervous system allows bidirectional communication through parasympathetic and sympathetic efferent and afferent neurons. There is compelling evidence that the enteric microbiota has considerable influence on this intricate system. Thus, potential targets for radionuclide imaging of the gut-brain axis comprise the autonomic nervous system and gut microbiota, including microbiota-specific substances.

Compositional changes in the gut microbiota in patients with Parkinson disease (PD) have been reported in several studies and may influence pathogenesis in several ways. For instance, curliproducing Escherichia coli bacteria have been documented to induce $\alpha$-synuclein aggregation in the gut (1). These aggregates may then spread via autonomic neurons to the central nervous system, including the substantia nigra (and many other structures), eventually causing PD (2). To our knowledge, no microbiotarelated imaging studies have been published, but validated methods to visualize the autonomic nervous system exist. We previously hypothesized that PD comprises two subtypes: one that originates in the enteric nervous system and spreads via the autonomic nervous system to the brain (body-first), and one that originates within the central nervous system and spreads via neuronal connections to other parts of the brain and the autonomic nervous system (brain-first). In this framework, the autonomic nervous system is affected at very different time points of the disease, depending on the subtype. Thus, radionuclide imaging of the autonomic nervous system may serve as an essential tool to obtain a detailed understanding of the temporal development of PD.

Parasympathetic innervation can be measured with the PET radiotracer 5 - ${ }^{11} \mathrm{C}$-methoxy-donepezil $\left({ }^{11} \mathrm{C}\right.$-donepezil), an acetylcholinesterase inhibitor. It is a reproducible method to quantify the cholinergic terminals in the gastrointestinal tract. Kinetic modeling of several internal organs shows a strong correlation between tracer volume of distribution and SUV (3). Therefore, a simple static acquisition $45 \mathrm{~min}$ after ${ }^{11} \mathrm{C}$-donepezil injection yields highquality images of gastrointestinal acetylcholinesterase density mirroring known cholinergic innervation (Fig. 1). The first comparative study in early-to-moderate PD patients showed a significantly decreased signal in the pancreas (22\% loss) and the small

Received May 25, 2021; revision accepted Jun. 29, 2021.

For correspondence or reprints, contact Per Borghammer (perborgh@rm.dk).

Published online July 22, 2021.

COPYRIGHT (C) 2021 by the Society of Nuclear Medicine and Molecular Imaging. intestines (35\% loss) (4). The colon was not investigated in that study. Next, in early-stage PD patients a loss of $22 \%$ was detected in the colon and $14 \%$ in the small intestine and renal cortex (5). In a study of patients with isolated rapid-eye-movement (REM) sleep behavior disorder, the strongest prodromal marker for $\mathrm{PD}$, the colon and small-intestine ${ }^{11} \mathrm{C}$-donepezil SUV was decreased to the same extent as in patients with PD (Fig. 1) (6). This finding firmly suggests that some PD patients (those who develop REM sleep behavior disorder before parkinsonism) exhibit severe autonomic denervation years before they receive a PD diagnosis. This hypothesis was tested in a recent study in which de novo PD patients were allocated to a brain-first group (without premotor REM sleep behavior disorder) and a body-first group (with premotor REM sleep behavior disorder) (7). Brain-first patients should, according to the hypothesis, have almost intact parasympathetic innervation of the colon. Conversely, body-first patients should exhibit severe parasympathetic denervation. Indeed, the body-first group had a significantly lower colon ${ }^{11} \mathrm{C}$-donepezil SUV than the brain-first group. Also, the brain-first group showed only minimally reduced SUVs compared with healthy controls (Fig. 1). This finding supports the hypothesis that PD consists of a brain-first subtype and a body-first subtype. Recently, the ${ }^{11} \mathrm{C}$-donepezil PET method was further validated by studying patients with bilateral vagotomy after esophageal cancer surgery. Such patients lack vagal cholinergic innervation of the abdominal organs, except pelvic organs and the descending colon. Indeed, the ${ }^{11} \mathrm{C}$-donepezil SUV was lower in both colon and small intestine in this patient group (Fig. 1) (8). Interestingly, the colonic SUV reduction was most prominent in the proximal part of the colon, mirroring the vagal parasympathetic projections. To summarize, ${ }^{11} \mathrm{C}$-donepezil PET is a reliable method to quantify acetylcholinesterase density, believed to reflect at least in part the parasympathetic innervation of the gastrointestinal tract.

Future studies of the parasympathetic nervous system should include ${ }^{18} \mathrm{~F}$-fluoroethoxybenzovesamicol, a promising PET tracer to evaluate the cholinergic system. ${ }^{18} \mathrm{~F}$-fluoroethoxybenzovesami$\mathrm{col}$ is a vesicular acetylcholine transporter ligand, which is a more specific cholinergic target than acetylcholinesterase. Thus, ${ }^{18} \mathrm{~F}-\mathrm{flu}-$ oroethoxybenzovesamicol may replace ${ }^{11} \mathrm{C}$-donepezil in future studies of gastrointestinal parasympathetic innervation.

Radionuclide imaging can also be used to assess functional abnormalities in the gastrointestinal tract. Up to $50 \%$ of $\mathrm{PD}$ patients have symptoms, most likely caused by gastric dysfunction. Nevertheless, studies using gastric emptying scintigraphy, the gold standard method for objective evaluation of gastric emptying time, have shown considerable heterogeneity and only a modest 


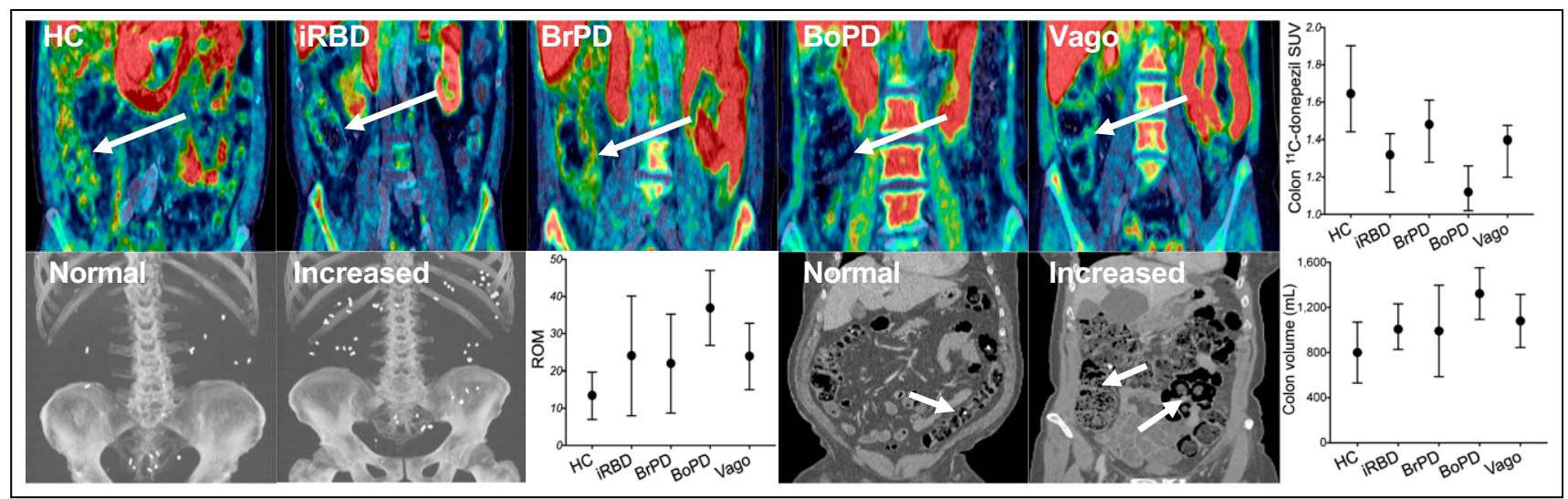

FIGURE 1. (Top) Representative ${ }^{11} \mathrm{C}$-donepezil SUV images of healthy control subject (HC), subject with isolated REM sleep behavior disorder (iRBD), brain-first PD patient (BrPD), body-first PD patient (BoPD), and vagotomized patient (Vago). Signal is high in HC and BrPD. Images are scaled from 0 to 3 SUV. Plot shows medians and interquartile ranges for ${ }^{11} \mathrm{C}$-donepezil data. (Bottom left) CT images of normal and increased number of retained radiopaque markers (ROMs) (i.e., normal and increased colon transit time). Plot shows medians and interquartile ranges for ROM data. (Bottom right) CT images of normal and increased colon volume. Plot shows medians and interquartile ranges for colon volume data.

delay in PD patients (9). Also, no robust association with subjective symptoms seems to exist (9).

Radionuclide imaging is often accompanied by a CT scan, from which additional important information can be obtained. Since constipation is one of the most frequently reported nonmotor symptoms in PD, several efforts have been made to objectively quantify the degree of colonic dysfunction. Colonic transit time (CTT) can be assessed by the radiopaque marker method, in which the number of retained markers can be counted on CT scans and a CTT estimate calculated (10). Also, total colon volume is easily obtained from even a low-dose CT scan and is considered another objective biomarker for colonic dysfunction. In support, CTT and colon volume are tightly correlated (10). Comparative studies have shown that patients with PD generally have a longer CTT and larger colon volume than healthy controls (10). Even patients with isolated REM sleep behavior disorder have a considerably prolonged CTT and increased colon volume (Fig. 1) (11). Interestingly, body-first PD patients exhibit significantly increased colon volume and CTT in comparison to brain-first PD patients. Collated, these studies illustrate that objective colonic dysfunction is present years before the PD diagnosis in some patients, whereas other patients develop colonic dysfunction after the PD diagnosis.

In summary, radionuclide imaging of the parasympathetic nervous system is a reproducible method to investigate autonomic degeneration in patients with PD. CT imaging provides additional information on gut dysfunction. Future radionuclide studies of the gut-brain axis in PD should explore new molecular targets, such as targets in the sympathetic nervous system, enteric nervous system, and microbiota-specific substances. Such studies will further elucidate the importance of the gut-brain axis in PD and other important disorders, including diabetic neuropathy.

\section{DISCLOSURE}

No potential conflict of interest relevant to this article was reported.

\section{REFERENCES}

1. Sampson TR, Challis C, Jain N, et al. A gut bacterial amyloid promotes alphasynuclein aggregation and motor impairment in mice. eLife. 2020;9:e53111.

2. Braak H, Rub U, Gai WP, Del Tredici K. Idiopathic Parkinson's disease: possible routes by which vulnerable neuronal types may be subject to neuroinvasion by an unknown pathogen. J Neural Transm. 2003;110:517-536.

3. Gjerløff T, Jakobsen S, Nahimi A, et al. In vivo imaging of human acetylcholinesterase density in peripheral organs using ${ }^{11} \mathrm{C}$-donepezil: dosimetry, biodistribution, and kinetic analyses. J Nucl Med. 2014;55:1818-1824.

4. Gjerløff T, Fedorova T, Knudsen K, et al. Imaging acetylcholinesterase density in peripheral organs in Parkinson's disease with ${ }^{11} \mathrm{C}$-donepezil PET. Brain. 2015;138: 653-663.

5. Fedorova TD, Seidelin LB, Knudsen K, et al. Decreased intestinal acetylcholinesterase in early Parkinson disease: an ${ }^{11} \mathrm{C}$-donepezil PET study. Neurology. 2017; 88:775-781.

6. Knudsen K, Fedorova TD, Hansen AK, et al. In-vivo staging of pathology in REM sleep behaviour disorder: a multimodality imaging case-control study. Lancet Neurol. 2018;17:618-628.

7. Horsager J, Andersen KB, Knudsen K, et al. Brain-first versus body-first Parkinson's disease: a multimodal imaging case-control study. Brain. 2020;143: 3077-3088.

8. Fedorova TD, Knudsen K, Hartmann B, et al. In vivo positron emission tomography imaging of decreased parasympathetic innervation in the gut of vagotomized patients. Neurogastroenterol Motil. 2020;32:e13759.

9. Knudsen K, Szwebs M, Hansen AK, Borghammer P. Gastric emptying in Parkinson's disease: a mini-review. Parkinsonism Relat Disord. 2018;55:18-25.

10. Knudsen K, Fedorova TD, Bekker AC, et al. Objective colonic dysfunction is far more prevalent than subjective constipation in Parkinson's disease: a colon transit and volume study. J Parkinsons Dis. 2017;7:359-367.

11. Knudsen K, Fedorova TD, Hansen AK, et al. Objective intestinal function in patients with idiopathic REM sleep behavior disorder. Parkinsonism Relat Disord. 2019;58:28-34 